\title{
Adenoid cystic carcinoma, report of a case
}

\begin{abstract}
Introduction: Adenoid cystic carcinoma (CAQ) is a typical tumor of the salivary glands and infrequent in the breast (less than $0.1 \%$ ). It stands out for being triple negative with a favorable prognosis. Its infrequency and slow progression mean that today there is no consensus regarding its therapeutic management and follow-up.
\end{abstract}

Objective: To provide a new case to learn more about the nature, management and evolution of this type of neoplasm.

Material and methods: We analyzed the data collected in our case and compared it with what was published in the literature.

Results: a typical triple negative case is presented, with a large size with respect to the breast (T2), so it was decided to perform a simple mastectomy as initial treatment with immediate reconstruction due to the absence of axillary involvement. Subsequently, it was decided not to apply adjuvant treatment since the tumor was not aggressive, the hormone receptors were negative, and the final lesion was less than $5 \mathrm{~cm}$ with wide margins and no evidence of lymphovascular invasion.

Conclusion: To date, breast CAQ continues to present uncertain management and followup given that we do not have enough data in the literature to know its evolution correctly, therefore more studies are needed with the intention of being able to standardize treatment and surveillance of this tumor.

Keywords: adenoid cystic carcinoma, triple negative, breast carcinoma, good prognosis, aggressive
Volume 12 Issue 3 - 202I

\section{Laura Bonilla García, Cristina López Beltrán, Aránzazu Maraví San Martín, Paola Trillo Martínez \\ Department of Gynecology and Obstetrics, Hospital Quiron Sagrado Corazón, Sevilla, Spain}

Correspondence: Laura Bonilla García, Department of Gynecology and Obstetrics, Hospital Quiron Sagrado Corazón, Calle camino Villanueva del Pitamo 6, Oficiales 2, pabellón 232. CP 4I013, Sevilla. Sevilla, Spain, Tel +34620822757, Email laura.b.g@hotmail.com

Received: April 22, 2021 | Published: May 13, 2021
Abbreviations: CAQ, adenoid cystic carcinoma; CIE, inferoexternal quadrant; MRI, magnetic resonance imaging; BAG

\section{Introduction}

The mammary gland and salivary glands are tubulo-acinar exocrine glands that can cause neoplasms with identical morphological characteristics, but with differences in incidence and clinical behavior. Adenoid cystic carcinoma (CAQ) is a typical tumor of the salivary glands, but it can appear in other organs such as the tracheo-bronchial tree, uterine cervix, larynx, Bartholin's gland and breast. ${ }^{1}$ It is a rare neoplasm in the breast. (less than $0.1 \%$ ). It is typically triple negative with a favorable prognosis. Its low frequency and its indolent course cause difficulties in the agreement of the evaluation and the therapeutic consensus, as well as in the use of the sentinel node technique. ${ }^{2}$

\section{Case report}

50 -year-old patient who consulted for the appearance of a painful nodule in the right breast of about 2 months of evolution. As a personal history, the following stand out: menarche at 12years of age, nulliparous, regular menstruation, smoker of 5-6 cigarettes a day. Ingestion of anovulatory drugs for less than a year at age 18. She does not report a history of breast cancer in the family or of another hereditary-familial neoplasm. On examination, a nodule in the inferoexternal quadrant (CIE) of the right breast was palpable, about $3-4 \mathrm{~cm}$ of solid consistency, well defined and not attached to deep planes. Rest of the breast without findings. Areola-nipple complex within normality. Axillary or supraclavicular lymphadenopathy is not palpable. In the mammary ultrasound it is visualized as a hypoechoic ovoid image with lobulated contours and approximate diameters of $27 \times 20 \mathrm{~mm}$. This image shows a solid ultrasound appearance, not seen in previous studies, showing suspicious ultrasound features. No other relevant findings. Nor are pathological lesions seen in the axillae, infraclavicular regions, or in the internal mammary chain. The lesion is classified as BIRADS-4a and a core needle biopsy of it is requested, obtaining 4 cylinders. The pathological study reports findings consistent with adenoid cystic carcinoma. An immunohistochemical study was performed that confirmed the biphasic nature of the lesion with positive ductal epithelial cells for cytokeratin 7 and ckit (CD 117) and myoepithelial cells positive for actin and P63. The Ki 67 proliferation index was positive in $15-20 \%$ of the cells. Estrogen receptors were less than $5 \%$, with weak intensity, and progesterone receptors and the HER2 study were negative. To assess the local extension, a bilateral breast resonance imaging (MRI) with and without contrast was performed, which describes a nodular lesion in ICD of the right breast with irregular lobulated margins, hypointense with multiple small cystic foci in its interior millimetric and presenting a maximum diameter of about $35 \times 20 \times 20 \mathrm{~mm}$ for its anteroposterior, transverse and craniocaudal diameters. At the axillary level, no findings compatible with lymphadenopathy were identified. After the administration of intravenous contrast, the nodular enhancement of the lobulated and irregular margins of the ICD of the right breast stands out, which present intense enhancement in the initial, early phase and a tendency to maintain the level of enhancement in the post-initial phase, forming a dynamic of the nonspecific plateau type 2 enhancement. The presence of some anterior and posterior spiculation depending on the fundamentally medial margin of the nodule stands out, so measuring the end of these enhancements the anteroposterior dimension would be $44 \mathrm{~mm}$. No other nodular enhancements or other non-mass enhancements suspected of malignancy. The MRI concluded the presence of a BIRADS 6 lesion compatible with adenoid cystic carcinoma that manifests as an irregular nodule in ICD of the right breast with the referred measures. Its lateral margin of about $11.4 \mathrm{~mm}$ is located at a distance from the skin and due to its location, in the 
mid-posterior third of the breast without involvement of the thoracic wall or extension to the nipple areola complex. Neither are there signs of multifocality or multicentricity. The case was presented to the committee of the breast pathology unit and a simple mastectomy was performed with a selective sentinel node biopsy, which was negative in the intraoperative study, so the surgery was completed with an immediate reconstruction carried out by surgery plastic. The pathological study of the mastectomy surgical piece showed a whitish, firm, stellate neoformation with poorly defined edges, $3.5 \mathrm{~cm}$ in diameter, located in the lower-internal quadrant, without contact with the skin and $1 \mathrm{~cm}$ from the pectoral resection margin. No presence of lymphovascular or perineural invasion. Free resection edges. Negative sentinel node: 0/1. Right breast: Pathological stage pT2 (m) pN0 (sn). After surgery, a reassessment of the case was carried out in the oncology clinic, not considering the need for the administration of neoadjuvant chemotherapy (low sensitivity), hormonal therapy (negative receptors) or radiotherapy (mastectomy with lesion less than $5 \mathrm{~cm}$, wide margins and no evidence of lymphovascular invasion).

\section{Discussion}

CAQ of the breast is a rare carcinoma, representing less than $0.1 \%$ of all malignant neoplasms in this area, with low malignant potential, in contrast to its analogue in the salivary gland, which is characterized by its high aggressiveness. It usually affects postmenopausal women, between the sixth and seventh decade of life, although there are also cases described in men..$^{3-5}$ The most frequent form of clinical presentation is that of a slow-growing, painful tumor with a slight predilection for the subareolar area and the upper external quadrants. Image characteristics are nonspecific and vary considerably. It is mainly unifocal, presenting in a multifocal way on rare occasions. In one of the most recent case series presented, Fernández de los Reyes et al., describe 12 cases with a mean age of 63.5 years and a single unilateral and multifocal case 2. The definitive diagnosis is the anatomopathological one, it is usually made by BAG5. In CAQ several growth patterns are distinguished: tubular, cribriform or solid. Histologically, it is composed of epithelial or luminal cells (CK7+, $\mathrm{TN})$ and myoepithelial cells (p63+), with glandular lumens filled with mucin (alcian blue + ) and pseudo-lights filled with stromal matrix $(\mathrm{CKIT}+)$, basal lamina and fibroblasts. The subtypes or grades are:

i. Classic, the most common, there may be squamous or sebaceous metaplasia, infiltrative border, differential diagnosis with collagenous spherulosis and tubular carcinoma;

ii. Solid-basaloid, basaloid cells with atypia and mitosis, necrosis, differential diagnosis with fat necrosis and non-special infiltrating carcinoma, and

\section{iii. With high-grade transformation. ${ }^{6}$}

Immunohistochemically, luminal cells are positive for CD117 and myoepithelial cells are positive for smooth muscle actin, calponin, and p63. CAQ is generally negative for estrogen receptors (ER), progesterone receptors (PR), and HER27. Although the histology of primary breast CAQ is identical to that of salivary gland tumors from another site (head and neck), have a less aggressive biological behavior (slow growth, little lymph node involvement and distance) and therefore a better prognosis, with disease-free survival figures close to $100 \%$ and overall survival around $85 \% 8$, respectively, provided that the criteria diagnoses are applied correctly and allow the identification of pure forms, that is, tumor components of another histological type do not coexist, in which case survival will depend on that other component. The second component usually corresponds to an infiltrating or ductal carcinoma in situ 9, type NOS (not otherwise specified). On the other hand, classic triple negative primary breast tumors are generally associated with a worse prognosis, while primary breast CAQ, despite being triple negative, has a more favorable clinical course. ${ }^{1,7}$ Arpino et al., ${ }^{8}$ in a review of 182 cases, found 14 cases with axillary involvement and 10 cases with distant metastases. Most of the metastases, also pulmonary, did not show initial axillary involvement. In CAQ surgery remains the main treatment focus. Multiple studies have published the risk of presenting positive margins after breastconserving surgery, with a relative impact on survival. ${ }^{8-10}$ Most studies recommend adjuvant radiotherapy to reduce local recurrence despite the fact that the role of radiotherapy in the survival of these patients has not been established. ${ }^{11,12}$ Chemotherapy is not used in a regulated manner due to the lack of comparative studies on the results with and without chemotherapy14. Regarding hormonal therapy, its use in these cases is not clear, but it could be used in cases of positive hormone receptors. Local recurrence is observed in 3-18\% of patients and survival at 10 years is greater than $90 \%{ }^{8,9,13-15}$ However, since studies regarding recurrence rates (local recurrence: $3-18 \%$ of patients) are scarce,,$^{11,13,15}$ it is difficult to establish the best method of follow-up of these patients. ${ }^{16}$

\section{Conclusion}

Most breast cancers with triple-negative basal-type breast characteristics are generally high-grade aggressive tumors. However, adenoid cystic carcinoma seems to show a phenotype similar to the triple negative baseline but with a low-grade pattern and a favorable clinical behavior. Furthermore, published data show a 10-year survival of $90 \%-100 \%$, a very low frequency of lymph node metastases, and a good response to surgical treatment. Despite this, we must be cautious with the results since, due to its low incidence, few complete studies have been carried out that reflect the characteristics of adenoid cystic breast carcinoma.

\section{Acknowledgments}

The authors thank the staff of Radiology, Nuclear Medicine, Pathology, Plastic Surgery and Oncology at Hospital Quiron for their collaboration.

\section{Funding}

None.

\section{Conflicts of interest}

The authors declare that they have no conflict of interest.

\section{References}

1. Cavanzo FJ, Taylor HB. Adenoid cystic carcinoma of the breast. Cancer. $1969 ; 4: 40-45$.

2. Fernández de los Reyes I, Córdoba Iturriagagoitia A, Chouza Sanin M, et al. Adenoid cystic carcinoma of the breast, 22 years of experience. Rev Esp Patol. 2020;53(4):206-212.

3. Lakhani SR, Ellis IO, Schnitt SJ et.al. WHO classification of tumours of the breast. Lyon: IARC; 2012.

4. McClenathan JH JH, de la Rosa G. Adenoyd cystic breast cancer. Am J Surg. 2002;183:646-649.

5. Bhosale SJ, Kshirsagar AY, Patil RK, et al. Adenoid cystic carcinoma of the male breast: A case report. Int J Surg Case Report. 2013;4:480-482.

6. Vasudev P, Onuma K. Secretory breast carcinoma unique, triple-negative carcinoma with a favorable prognosis and characteristic molecular expression. Arch Pathol Lab Med. 2011;135(12):1606-1610. 
7. Vranic S, Bender R, Palazzo J, et al. A review of the adenoid cystic carcinoma of the breast with emphasis on its molecular and genetic characteristic. Hum Pathol. 2013;44:301-309.

8. Arpino G, Clark GM, Mohsin S, et al. Adenoid cystic carcinoma of the breast: molecular markers, treatment and clinical outcome. Cancer. 2002;94:1-27.

9. Khanfir K, Kallel A, Villette S, et al. Management of adenoid cys- tic carcinoma of the breast: a Rare Cancer Network study. Int J Radiat Oncol Biol Phys. 2012;82:2118-2124.

10. Millar BM, Kerba M, Youngson B, et al. The potential role of breas conservation surgery and adjuvant breast radiation for adenoid cystic carcinoma of the breast. Breast Cancer Res Treat. 2004;87:225-232.

11. Kulkarni N, Pezzi CM, Greif JM, et al. Rare breast cancer: 933 adenoid cystic carcinomas from the National Cancer Data Base. Ann Surg Oncol. 2013;20:2236-2241.
12. Franzese C, Zei G, Masoni T, et al. Adenoid cystic carcinoma of the breast. The double face of an exocrine gland carcinoma. Strahlenther Onkol. 2013;189:1049-1050.

13. Cadoo KA, McArdle O, O'Shea A-M, et al. Management of unusual histological types of breast cancer. Oncologist. 2012;17:1135-1145.

14. Li N, Xu L, Zhao H, et al. A comparison of the demographics, clinical features, and survival of patients with adenoid cystic carcinoma of major and minor salivary glands versus less common sites within the surveillance, epidemiology, and end results registry. Cancer. 2012;118:3945-3953.

15. Ghabach B, Anderson WF, Curtis RE, et al. Adenoid cystic carcinoma of the breast in the Uni- ted States (1977-2006): a population-based cohort study. Breast Cancer Res. 2010;12:R54.

16. Pérez JA, Pobrete MT, Deichier F, et al. Adenoid cystic breast carcinoma. Reporte de un caso Cuad Cir. 2006;20:70-74. 